\title{
Development of a New Scanning Ion Microbeam Analysis and Imaging Technique: Ion-Luminescence Microscopic Imaging and Spectroscopy (ILUMIS)
}

\author{
Wataru Kada ${ }^{* 1}$, Takahiro Satoh ${ }^{2}$, Akihito Yokoyama ${ }^{2}$, Masashi Koka ${ }^{2}$, and Tomihiro Kamiya ${ }^{2}$ \\ ${ }^{* 1}$ Gunma University, 1-5-1 Tenjincho, Kiryu, 375-8515 Gunma, Japan. \\ Fax: 81-277-30-1793, e-mail: kada.wataru@jaea.go.jp \\ ${ }^{2}$ Japan Atomic Energy Agency, 1233 Watanuki, Takasaki, 370-1292 Gunma, Japan.
}

\begin{abstract}
A new microscopic imaging technique using a $\mathrm{MeV}$ ion microbeam probe was demonstrated for the nondestructive imaging and spectroscopy analysis of micrometer-sized targets. Visible luminescence caused from the impact of $\mathrm{MeV}$ ion irradiation was analyzed using a newly developed Ion-Luminescence (IL) Microscopic Imaging and Spectroscopy (ILUMIS) system based on a microbeam line of a 3 MV single-ended accelerator. Wavelength-dispersive images of IL were obtained from particulate targets with a spatial resolution of $1 \mu \mathrm{m}$ and a wavelength resolution of $2 \mathrm{~nm}$. Particular chemical state inside the target was successfully obtained through the spectroscopy of IL analysis.
\end{abstract}

Key words: Ion luminescence, microbeam, microscopic imaging, chemical state

\section{INTRODUCTION}

Huge efforts are being spent to reduce and control potential environmental pollutants emitted through industrial activities, in an attempt to sustain human society. Several strict statements by the EU already prohibit transportation and use of certain heavy metals such as lead and arsenic [1]. As shown in the statement by REACH (Registration, Evaluation, Authorization and Restriction of Chemicals), the regulation takes its concept into the next stage to regulate the chemical states of toxic elements and manage the total risks for human bodies. As needs for, and variations of, chemical analysis have increased, so has the sensitivity and efficiency expected for newly developed analytical techniques.

Because most environmental analysis targets have only very little gross amount (ppb or ppm), Ion Beam Analysis (IBA) is one of the most effective tools for such precise analysis applications [2]. In-air micro Particle Induced X-ray Emission (PIXE) analyzes the elemental distribution of material at high sensitivity and efficiency by placing the target outside the vacuum chamber [3]. PIXE is applicable to the analysis of organic and inorganic targets polluted with harmful heavy elements by visualizing the elemental distribution of those with a detection level of some parts per million. The distribution of light elements (i.e., fluorine) can also be obtained with the same system in combination with Particle Induced Gamma-ray Emission (PIGE) [4].

However, these techniques have difficulties in revealing the distribution of the chemical states in a target, because the signals (characteristic $\mathrm{X}$-rays and gamma-rays) are normally insensitive to the chemical form of the host materials. To obtain an image of the chemical states (compounds) of a sample, a new IBA technique must be developed.

Recently, several papers have reported the potential of new IBA techniques for chemical-state analysis using photons induced by $\mathrm{MeV}$ ion impacts [5]. These ion-beam-induced photons originate from the interactions of the ion beam impacting with the outer-shell electrons and thus possess information on the chemical binding of target atoms. The energies of these photons range from several electron-volts, which corresponds to the wavelength from Ultra-Violet (UV), through visible (Vis), to Infra-Red (IR). The analysis technique that uses these photons is called Ion-Luminescence or Iono-luminescnce (IL) [6]. Spectroscopy and imaging of IL was studied for long time but high background noises prevent actual application of these techniques $[7,8]$.

In this study, a prototype of an IL analysis and imaging system using an ion microbeam has been developed on an existing ion microbeam system with noise-reduced photon-counting. The existing system was combined with an in-air micro-PIXE analysis system; thus it can obtain chemical-state imaging from the same areas of interest of elemental distribution as taken by micro-PIXE. Panchromatic and monochromatic IL images were created for particulate targets.

\section{MATERIALS AND METHODS}

2.1 IL Analysis System

A new IL analysis system was developed on a microbeam line of a $3 \mathrm{MV}$ single-ended accelerator at the ion-irradiation research facility TIARA (Takasaki Ion Accelerators for Advanced Radiation Application), Japan Atomic Energy Agency [9].

Figure 1 shows a schematic drawing of the Ion Luminescence Microscopic Imaging and Spectroscopy (ILUMIS) analysis system combined with in-air micro PIXE. A Si(Li) detector (PGT LS30135) was already mounted on the microbeam chamber at an angle of $40^{\circ}$ to the beam axis. Optics for IL was installed into same system in the symmetry position of the $\mathrm{Si}(\mathrm{Li}) \mathrm{X}$-ray detector with respect to the beam axis. An aspheric lens (Ocean Optics 84-UV-25) was coupled with a 1000 $\mu \mathrm{m} \Phi$ optical fiber feed-through via an SMA connector. 


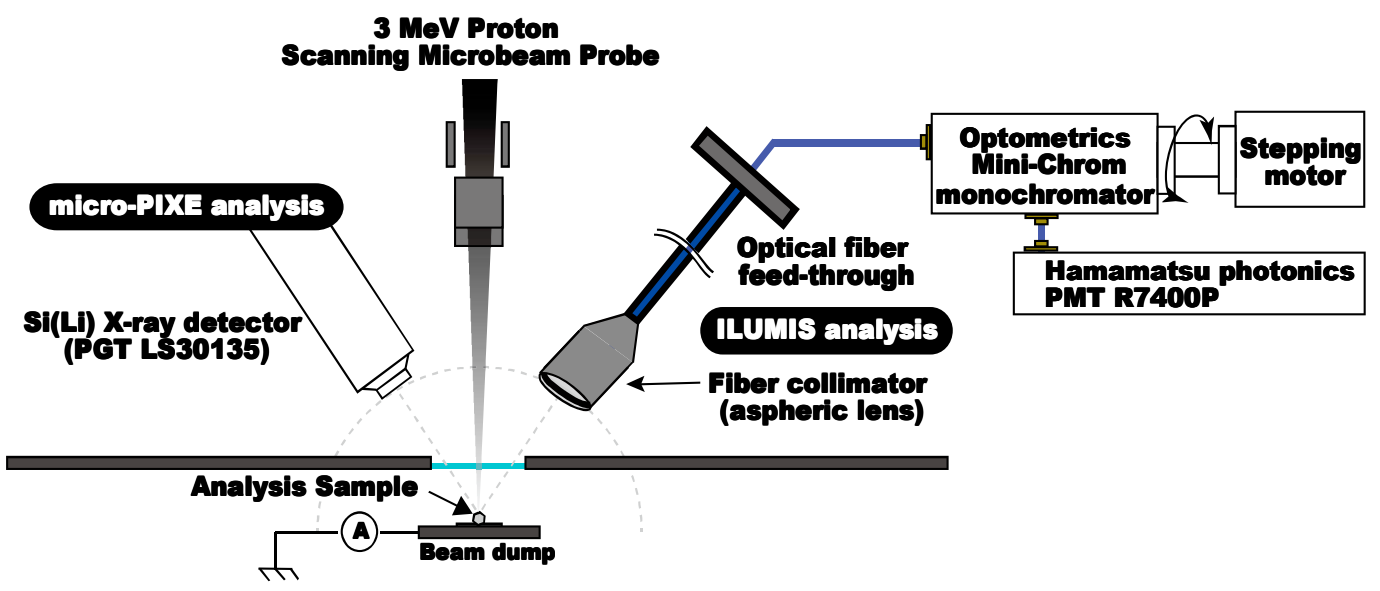

Fig. 1. Schematic drawing of an analysis system of

Ion Luminescence Microscopic Imaging and Spectroscopy (ILUMIS) combined with an in-air micro PIXE system.

Distance from the lens to the sample was determined as $100 \mathrm{~mm}$. A Photo-Multiplier Tube (PMT; Hamamatsu Photonics R7400P) was connected to the output of the optical fiber feed-through via a monochromator Mini-Chrom to obtain monochromatic IL imaging and spectrum. This PMT is sensitive to IL photons in the wavelength region from 300 to $650 \mathrm{~nm}$. The output of PMT was connected to a charge-sensitive amplifier and a shaping amplifier before being delivered into the data acquisition system. To combine the data obtained from the two microbeam analysis methods, the same signal processing system as for the micro-PIXE [10] was used for the IL analysis.

Entire vacuum chamber was placed inside a dark room to reject stray light; thus only photons emitted from ion impacts were observed by the installed photon-counting units. Negative high voltage of $-800 \mathrm{~V}$ was applied to the PMT R7400P to collect photon counts, observed with a Multi-Channel Analyzer (MCA). Background noise was observed as being approximately $100 \mathrm{cps}$ in the dark room at room temperature. This count rate was reduced to around $10 \mathrm{cps}$ by cooling the PMT to around $0{ }^{\circ} \mathrm{C}$. This reduction was quite effective in gaining a high $\mathrm{S} / \mathrm{N}$ ratio in the measurement of weak IL signals from the target [11].

\subsection{Signal Procedure}

Figure 2 shows example of spectra and images of a cell (Paramecium bursaria) target: (a) is an example of characteristic X-ray spectrum, while photon count distribution of IL was shown in (b). Microscopic Image of PIXE (c) and IL (d) were also shown in the figure. A semiconductor X-ray detector was used to obtain a spectrum for the PIXE analysis, in which higher energy resolution can extract information about the energy of each characteristic X-ray as $\mathrm{P} \mathrm{K}_{\alpha}$ and $\mathrm{S} \mathrm{K}_{\alpha}$ in the example. On the other hand, the IL spectrum takes the shape of a much broader curve. While PMT can obtain the total photon count with high accuracy, the pulse height of the signals does not relate to the wavelength (energy) of the photons.

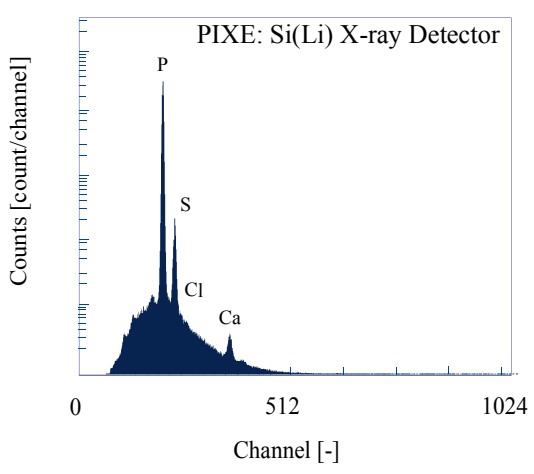

(a) Example of PIXE Spectrum

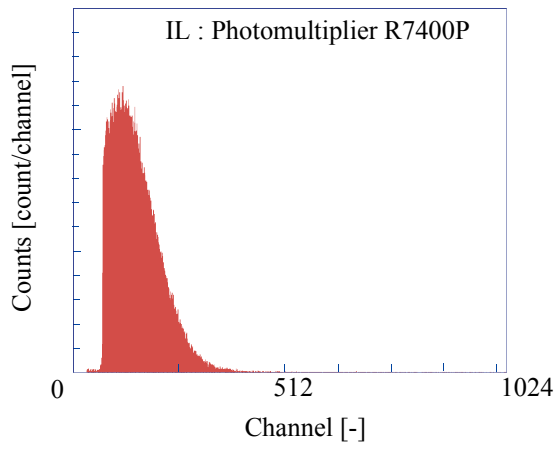

(b) Example of IBIL Spectrum

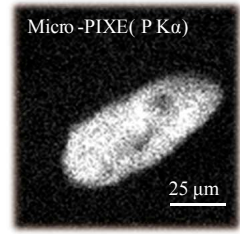

(c) Example of micro-PIXE distribution of $\mathrm{P} \mathrm{K}$

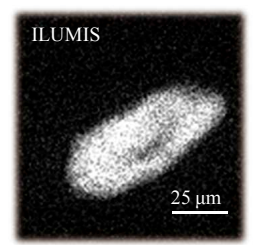

(d) Example of panchromatic IL distribution

Fig. 2. Examples of (a) PIXE spectrum and (b) IL spectrum.

(c) Elemental Distribution image of $\mathrm{P} \mathrm{K}_{\alpha}$ and (d) Panchromatic IL image of a cell (Paramecium bursaria) taken by PIXE / ILUMIS combined analysis. 


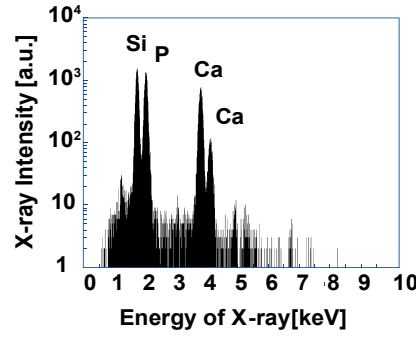

(a) PIXE Spectrum

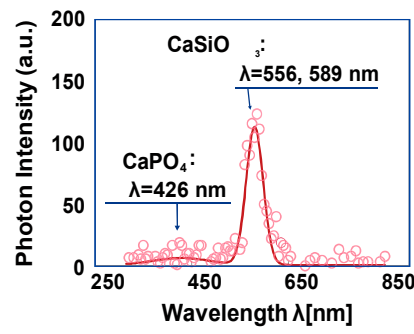

(b) IL Spectrum

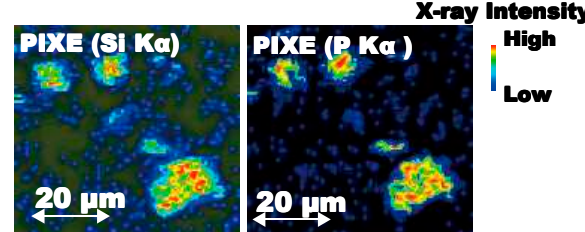

(c) Elemental Distribution by micro-PIXE

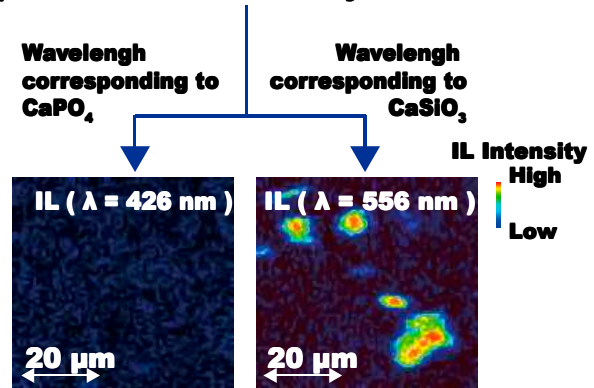

(d) Compound Distribution by ILUMIS

Fig. 3. Example of (a) PIXE and (b) IL spectrum obtained from aerosol targets.

(c) Elemental distribution of the same aerosol particles were analyzed by micro-PIXE, while

(d) chemical compound of the same aerosol particles were also obtained by ILUMIS.

Thus it was necessary to install the monochromator in front of the photon detector to observe the wavelength dispersive IL spectrum. The example micro-PIXE image shown in Fig. 2(c) indicates the phosphorus distribution, which roughly gives the structure of the cell. In contrast, the example IL image shown in Fig. 2(d) is panchromatic at this stage. These two images give similar distributions. This result signifies that the newly developed IL system can provide 2-D images with spatial resolution similar to that of micro-PIXE analysis. It is also clear that this IL analysis is applicable not only to inorganic targets but also to organic ones.

\subsection{Evaluation of Optics for IL}

An experiment to evaluate ILUMIS analysis was performed on several uniform standard targets, such as scintillator and insulators. First, ZnS:Ag film was irradiated with the $3 \mathrm{MeV}$ proton microbeam with a beam current of under $0.1 \mathrm{pA}$ and a beam-scanning area of $100 \times 100 \mu^{2}$. A high count rate (>100,000 photons) was observed from $\mathrm{ZnS}: \mathrm{Ag}$, with a clear peak around $450 \mathrm{~nm}$ corresponding to the luminescence center of $\mathrm{ZnS}: \mathrm{Ag}$. IL spectra were also successfully obtained from other scintillators [i.e., YAG:Ce $(\lambda: 550 \mathrm{~nm})$ and $\left.\mathrm{CaF}_{2}: \mathrm{Eu}(\lambda: 435 \mathrm{~nm})\right]$ and several insulators like alpha- $\mathrm{Al}_{2} \mathrm{O}_{3}(\lambda: 326 \mathrm{~nm}$ and $415 \mathrm{~nm})$. It was confirmed that the developed system had responded well to IL signals at the specific wavelength $(300-850 \mathrm{~nm})$.

\section{RESULTS AND DISCUSSION}

3.1 Microscopic Analysis of IL

IL analysis of particulate aerosols was demonstrated to sense the chemical structures of microscopic targets. Airborne particles were sampled at the Takasaki
Institute of JAEA from October to December 2010. The particles were fixed on the carbon film to be irradiated by $3 \mathrm{MeV} \mathrm{H}^{+}$with a current of approximately $100 \mathrm{pA}$. The images were obtained by beam scanning with an area of $50 \times 50 \mu \mathrm{m}^{2}$. Fig. 3 shows IL/PIXE spectra and images of the particulate aerosols. The characteristic $1.74 \mathrm{keV} \mathrm{Si} \mathrm{K}_{\alpha}$ X-ray was strongly evident in the PIXE spectrum shown in Fig. 3(a), with several peaks corresponding to various other elements such as phosphorous and calcium. Distributions of IL at several wavelengths were also given from the same aerosols, as shown in Fig. 3(b), as a form of the spectrum. Two characteristic X-ray distributions of silicon and phosphor were shown in Fig. 3(c). It was not clearly distinguishable from the difference in these distributions as to which element is a major element in the particles. On the contrary, Fig. 3(d) shows monochromatic IL imaging; strong luminescence at $556 \mathrm{~nm}$ was obtained from each particle, while almost no IL count was observed from other wavelengths. According to the database of luminescence peaks [12], the chemical compound of these aerosols might be identified as $\mathrm{CaSiO}_{3}$ from the peak wavelengths $[13,14]$.

\subsection{Microscopic Imaging by IL}

Microscopic imaging by IL was also performed with a proton microbeam at a current of approximately $200 \mathrm{pA}$ and a scanning area of $800 \times 800 \mu \mathrm{m}^{2}$. Standard samples of three asbestoses [(a) chrysotile $\left(\mathrm{Mg}_{6} \mathrm{Si}_{4} \mathrm{O}_{10}(\mathrm{OH})_{8}\right)$, (b) crocidolite $\left(\mathrm{Na}_{2}\left(\mathrm{Fe}^{2+}{ }_{3} \mathrm{Fe}^{3+}{ }_{2}\right) \mathrm{Si}_{8} \mathrm{O}_{22}(\mathrm{OH})_{2}\right)$, and (c) amosite $\left.\left(\mathrm{Mg}_{7} \mathrm{Si}_{8} \mathrm{O}_{22}(\mathrm{OH})_{2}, \quad \mathrm{Fe}^{2+}{ }_{7} \mathrm{Si}_{8} \mathrm{O}_{22}(\mathrm{OH})_{2}\right)\right]$ were prepared on thin carbon plate. Fig. 4 shows an example of spectra and images of asbestos samples by PIXE and ILUMIS analysis. 


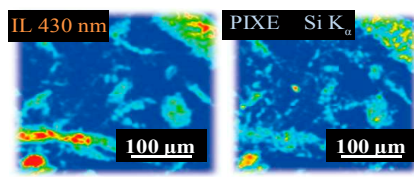

(a) chrysotile sample

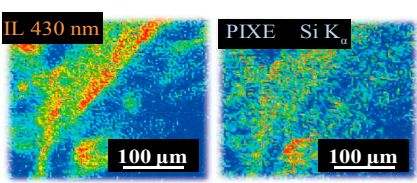

(b) crocidolite sample

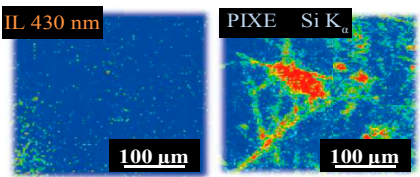

(c) amosite sample

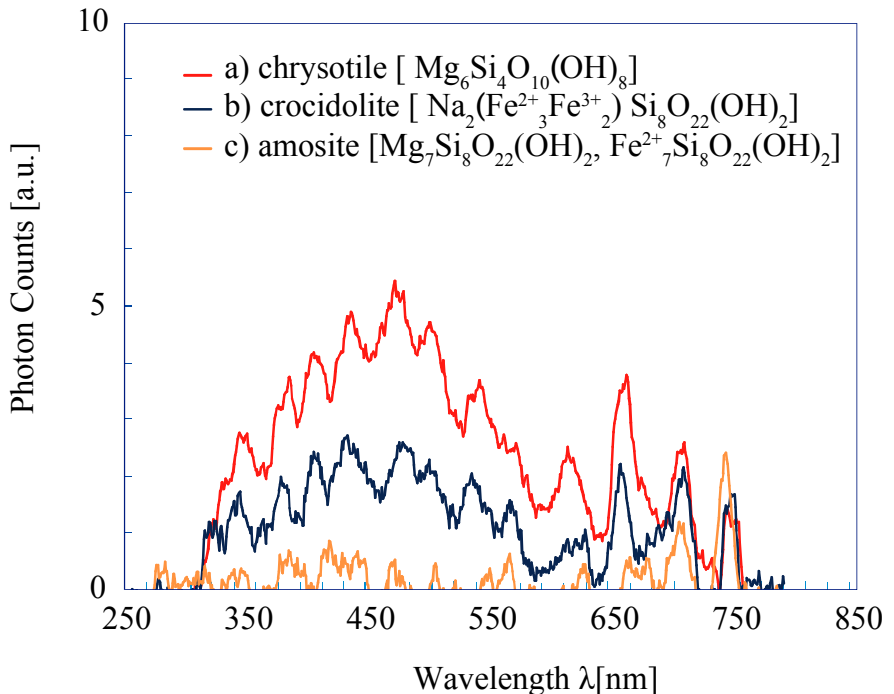

Wavelength $\lambda[\mathrm{nm}]$

Fig. 4. (left) ILUMIS and micro-PIXE imaging of three asbestoses: (a) chrysotile, (b) crocidolite, and (c) amosite. (right) IL spectra of (a) chrysotile, (b) crocidolite, and (c) amosite.

Strong peaks of $\mathrm{Si} \mathrm{K}_{\alpha}$ and $\mathrm{Mg} \mathrm{K}_{\alpha}$ was obtained in PIXE analysis, while $\mathrm{Fe} \mathrm{K}_{\alpha}$ was detected in two samples. Irons in specific chemical states as $\mathrm{Fe}^{2+}, \mathrm{Fe}^{3+}$ are also contained in crocidolite and amosite. IL images were also observed from each sample of asbestos, as shown on the right side of Fig. 4. A relatively low count rate of photons (around $100 \mathrm{cps}$ on average) was obtained from each asbestos sample. The major chemical compounds of the asbestos were silicon oxide and magnesium oxide, which mainly agreed with the luminescence peak of IL $[15,16]$. ILUMIS imaging of chrysotile well represents the image of that by PIXE Si $\mathrm{K}_{\alpha}$ X-rays. The count rate of IL became lower for the target crocidolite as shown in decreased intensity of the IL spectrum shown in Fig. 4. When amosite was irradiated by the same microbeam probe, almost no photons of IL were observed. PIXE analysis could obtain the elemental distribution from the targets; thus only the IL photons were not emitted from the sample. The result demonstrates that IL is strongly affected by the specific chemical states of iron and other rare elements that cannot be obtained from PIXE analysis.

\section{CONCLUSIONS}

A prototype IL spectroscopy and imaging system using an ion microbeam was newly designed and developed for the chemical state analysis of individual micrometer-sized targets. It was found that IL imaging was clearly obtained from several samples of cells, aerosols, and asbestos by $3 \mathrm{MeV}$ proton microbeam irradiations. IL spectroscopy also revealed the chemical compounds of aerosol particles from certain IL wavelengths. Further development is required to improve the efficiency of the optics for IL analysis, especially for observation of specific microscopic targets like asbestos, in which particular elements would affect IL analysis results. Quantitative assessments should be performed on the practical use of ILUMIS analysis.
Acknowledgment

This research was supported by MEXT/JSPS Grant-in-Aid for Young Scientists (B) 24710097 and a JAEA Grant-in-Aid for Exploratory Research.

References

[1] Directive 2002/95/EC of the European parliament and of the council on the restriction of the use of certain hazardous substances in electrical and electronic equipment, Official J. Europ. Union, L37 (2003) 19-23.

[2] M. Kasahara et al., Nucl. Instr. and Meth. B, 181, 1-4 (2001) 622-627.

[3] K. Ishii et al., Nucl. Instr. and Meth.B, 181, 1-4 (2001) 448-453.

[4] F. Gerab, et al, Nucl. Instr. and Meth.B, 136-138, (1998) 955-960.

[5] P.D. Townsend et al., Surf. Coat. Technol., 201,19-20 (2007) 8160-8164.

[6] K.G. Malmqvist, et al., Nucl. Instr. and Meth.B, 109-110 (1996) 227-233.

[7] C. Yang et al., Nucl. Instr. and Meth.B, 77, 1-4 (1993) 188-194.

[8] F. Watt et al., Nucl. Instr. and Meth.B 267, 12-13 (2009), 2113-2116.

[9] T. Kamiya, et al., Nucl. Instr. and Meth.B, 104, 1-4 (1995) 43-48.

[10] T. Sakai et al., Nucl. Instr. and Meth.B, 190, 1-4 (2002) 271-275.

[11] W. Kada, et al., Int. J. of PIXE, 21,1-2, (2011) 1-11.

[12]C. M. MacRae et al., Microscopy and Microanalysis, 14 (2008) 184-204.

[13] P. Dorenbos, J. Phys. Condens. Matter.,15 (2003) 2645-2665.

[14] F.C. Palilla,et al., J. Electrochem. Soc.. 117 (1970) 87-91.

[15]Y. Hibino et al., J. Non-Cryst. Solids,107,1 (1988) 23-26.

[16] T. Inokuma et al., J. Lumin. 80, 1-4 (1998) 247-251.

(Received February 18, 2013; Accepted June 13, 2013) 